Características clínicas, radiológicas y de laboratorio en niños con diagnóstico de

COVID-19: Metaanálisis de proporción única.

Clinical, radiological and laboratory characteristics in children diagnosed with

COVID-19: Meta-analysis of a single proportion.

\title{
AUTORES:
}

\section{Santiago Vasco-Morales}

Doctor en Medicina General y Cirugía. Especialista en Pediatría. Docente, Carrera de Obstetricia, Facultad de Ciencias Médicas, Universidad Central del Ecuador, Quito, Ecuador. Doctorando, Facultad de Medicina de Riberāo-Preto, Universidad de São Paulo, São Paulo, Brasil.

https://orcid.org/0000-0002-1370-9700

\section{Cristhian Vasco-Toapanta}

Estudiante, Colegio de Ciencias de la Salud, Universidad San Francisco de Quito, Quito, Ecuador.

https://orcid.org/0000-0002-2874-9836

\section{Paola Toapanta-Pinta}

Doctora en Medicina General y Cirugía. Especialista en Medicina Familiar. Docente, Carrera de Obstetricia, Facultad de Ciencias Médicas, Universidad Central del Ecuador, Quito, Ecuador. Doctorando, Facultad de Medicina de Riberāo-Preto, Universidad de São Paulo, São Paulo, Brasil. https://orcid.org/0000-0003-2804-2504

Dirección autor responsable: Iquique N14-121 y Sodiro - Itchimbía, Sector El Dorado. Teléfono: (593) 0985686993

Correo electrónico: $\underline{\text { snvasco@uce.edu.ec }}$ 


\section{Resumen}

Objetivo: Resumir la mejor evidencia disponible acerca de la frecuencia de presentación de: signos, síntomas, principales exámenes de laboratorio y, alteraciones radiológicas en pacientes menores de 19 años con diagnóstico de COVID-19.

Método: Mediante búsquedas en PubMed, Scopus y Google académico, se recopilaron los datos de los estudios que presentaban las características clínicas, de laboratorio y radiología, para realizar metaanálisis de proporción única.

Resultados: Se encontró 11 estudios, que incluían un total de 1180 pacientes. El metaanálisis presentó una mayor afectación del sexo masculino 0.56(IC 95\%; 0.51:0.61), pacientes asintomáticos 0.15(IC 95\%; 0.09:0.22), infecciones leves y muy leves 0.44(IC 95\%; 0.25:0.63), infecciones moderadas 0.44(IC 95\%; 0.38:0.45), incidencia de fiebre 0.56(IC 95\%; 0.48:0.65), tos 0.46(IC 95\%; 0.39:0.53), faringitis 0.18(IC 95\%; 0.03:0.39), en los exámenes de laboratorio presentó mayor incidencia la leucopenia 0.33(IC 95\%; 0.25:0.42), la Proteína C reactiva 0.19(IC 95\%; 0.15:0.24) y la Procalcitonina 0.26(IC 95\%; 0.16:0.46), los pacientes con alteración radiológica 0.68(IC 95\%; 0.56:0.79), pacientes graves $n=5$ y fallecidos $n=1$.

Conclusiones: La presencia de anormalidades radiológicas es mas frecuente que los signos clínicos de tos y fiebre que son los síntomas más comunes. En el hemograma: la leucopenia es mas frecuente. Existe una tendencia de mayor afectación en el sexo masculino. Este metaanálisis servirá de base para futuras comparaciones acerca de las similitudes o diferencias que pueden presentarse ahora que la pandemia está en América. También presenta limitaciones por lo que los resultados deben ser contrastados, con estudios prospectivos controlados, con mayor número de pacientes y un diseño mas estricto.

Palabras clave: niños, COVID-19, metaanálisis, diagnóstico 


\section{Abstract}

Objective: To summarize the best available evidence about the frequency of presentation of: signs, symptoms, main laboratory tests and, radiological alterations in patients younger than 19 years with a diagnosis of COVID-19.

Method: By searching PubMed, Scopus and academic Google, data from studies where present clinical, laboratory and radiological characteristics were collected to perform single-ratio meta-analyzes.

Results: 11 studies were found, including a total of 1180 patients. The meta-analysis showed a greater incidence of the male sex 0.56 (95\% CI; $0.51: 0.61)$, asymptomatic patients 0.15 (95\% CI; 0.09: 0.22), mild and very mild infections 0.44 (95\% CI; 0.25 : 0.63 ), moderate infections 0.44 (95\% CI; 0.38: 0.45), incidence of fever 0.56 (95\% CI; 0.48: 0.65), cough 0.46 (95\% CI; 0.39: 0.53), pharyngitis 0.18 (95\% CI; 0.03: 0.39 ), laboratory tests showed a higher incidence of leukopenia 0.33 (95\% CI; 0.25: 0.42), Creactive Protein 0.19 (95\% CI; 0.15: 0.24) and Procalcitonin 0.26 (95\% CI; 0.16: 0.46), patients with radiological alteration 0.68 (95\% CI; 0.56: 0.79), severe patients $n=5$ and deceased $n=1$.

Conclusions: The presence of radiological abnormalities is more frequent than the clinical signs of cough and fever, which are the most common symptoms. On the blood count: leukopenia is more frequent. There is a trend of greater incidence in the male sex. This meta-analysis will serve as a basis for future comparisons of the similarities or differences that may occur now that the pandemic is in America. It also has limitations so the results must be contrasted, with controlled prospective studies, with a greater number of patients and a stricter design.

Key words: children, COVID-19, meta-analysis, diagnosis 


\section{Introducción}

La pandemia de COVID-19 mantiene en alerta al sistema de salud mundial, con mayor afectación en los adultos ${ }^{1}$, al momento existe un amplio panorama acerca de la presentación clínica, de laboratorio y gabinete en este grupo. Sin embargo, la población pediátrica por sus características y vulnerabilidad propia de la edad amerita que se determine su perfil clínico-epidemiológico.

Está establecido que las características anatómicas, fisiológicas e inmunológicas de los niños influyen en el comportamiento y la epidemiología de las infecciones respiratorias, que en general son mas comunes a esta edad y su incidencia es inversamente proporcional a la edad del niño. En cuanto al sistema inmunológico destacan la inmadurez de la microbiota, sistema de complemento disminuido, reserva de neutrófilos baja, capacidad de fagocitosis y quimiotaxis disminuidas, además el menor funcionamiento de células NK condiciones que varían según la edad del niño ${ }^{2}$. Se debe considerar que después del nacimiento ocurre un gran desarrollo del parénquima pulmonar por formación de alveolos y la maduración de la microvasculatura hasta los 2-3 años, mientras que entre los 2 y 8 años se produce un aumento tanto del número como del tamaño de los alveolos. ${ }^{3}$ También es importante anotar que los niños y jóvenes tiene mayor expresión del receptor ECA2 en relación con los adultos ${ }^{4}$, todas estas características podrían explicar los diferentes patrones de presentación clínica en los pacientes afectados por esta enfermedad.

Para comprender la dinámica de la infección por COVID-19 en pacientes pediátricos, se buscó la literatura actual mas relevante y se realizó un metaanálisis de proporción única, con el objetivo de determinar la frecuencia con la que se presentan las características clínicas, radiológicas y de laboratorio en niños con esta infección.

\section{Material y Métodos}




\section{Estrategia de Búsqueda}

Se realizó la búsqueda en bases de datos disponibles en línea y que fueron publicados desde octubre del 2019 hasta el 19 de marzo 2020. Para la búsqueda de los artículos se examinó las Bases de datos: PubMed, Scopus y Google académico, se utilizó las palabras clave “2019 novel coronavirus disease”, "2019 novel coronavirus infection”, “2019nCoV disease", “2019-nCoV infection”, “coronavirus disease 2019”, “coronavirus disease-19", and" Child”, "Children”, "Pediatrics", "Infant”, "Infants”, sin restricción de idioma.

\section{Criterios de Inclusión}

Estudios observacionales, transversales, series de casos que presentaron 10 o más pacientes, donde se incluyen las características clínicas, radiológicas y laboratorio, de pacientes menores de 19 años con diagnóstico confirmado de COVID-19.

\section{Criterios de exclusión}

Artículos duplicados, que presentaban menos de 10 pacientes, artículos que sólo presentaban resumen, artículos retractados.

\section{Procedimiento de extracción de los datos}

Inicialmente se seleccionó los artículos de acuerdo a la lectura del resumen y se aplicó los criterios de inclusión y exclusión. Los trabajos incluidos fueron en consenso entre los autores. Por la diversidad de las publicaciones y la diferente forma de clasificar a los pacientes o los registros, se incluyó los datos que fueron recolectados en un formato similar al tomado como base, o a su vez fueron compilados desde el texto.

\section{Análisis estadístico}


Los estudios incluidos en los metaanálisis de proporcion única son observacionales y no comparativos (brazo único). Para este metaanálisis se utilizó el programa estadístico $\mathrm{R}$ versión $3.6 .1^{5}$ paquete meta $^{6}$, que proporciona métodos para el metaanálisis de efectos fijos y efectos aleatorios de proporciones individuales para calcular una proporción general, utilizando el método de varianza inversa y el modelo mixto lineal generalizado (GLMM).Existen 3 métodos estadísticos para combinar los estudios: a) el modelo de efectos fijos, b) modelo de efectos aleatorios y c) el modelo bayesiano. En el modelo de efectos fijos los estudios se combinan suponiendo que no hay heterogeneidad entre ellos, entonces todos construyen estimaciones de un efecto real, de tal manera que los estudios con muestras mayores tienden a contribuir más a la media ponderada. En el modelo de efectos aleatorios la inferencia se sustenta en suponer que los estudios incluidos en el análisis son una muestra aleatoria del universo de posibles estudios, y por lo tanto sus resultados son más conservadores ya que consideran una fuente extra de variación: la existente dentro de los estudios y la variación entre los estudios.

El programa $\mathrm{R}$ también estima la heterogeneidad. Los estudios heterogéneos se consideran robustos para realizar metaanálisis, normalmente siempre existirá algún grado de heterogeneidad y ésta puede presentar valores de $0 \%$ a $100 \%$, cuando el valor es $0 \%$ indica que es causado por un error de muestreo y no hay nada que explicar; cuando es igual al $100 \%$, la heterogeneidad puede explicarse por las verdaderas diferencias entre los estudios exclusivamente, se considera una heterogeneidad máxima tolerables hasta un 75\%. Además, presenta el valor p de la prueba de heterogeneidad (hipótesis nula: no hay heterogeneidad).

Finalmente, en metaanálisis es importante cuantificar el sesgo de publicación. El método mas aceptado es la prueba de regresión linear de Egger, el valor p que sugiere presencia de sesgo es: $<0,1$. 


\section{Consideraciones éticas}

Para este estudio se utilizó información de pacientes no identificados de bases de datos disponibles públicamente, por lo que no se obtuvo el consentimiento del paciente y no se requirió aprobación ética, por el contrario, se indica la autoría original de los artículos incluidos en el metaanálisis para permitir la confirmación de los datos.

\section{Resultados}

\section{Inclusión de los estudios}

Se encontró 11 estudios que describían las variables de interés en 1180 pacientes. El flujograma de selección de estudios se presenta en la figura No 1. La distribución de los pacientes por edad y sexo en cada estudio se resumen en la tabla No.1, el resumen de los datos clínicos de laboratorio y radiología se presentan en la tabla No.2.

\section{Resultados del Metaanálisis, heterogeneidad y sesgo de publicación}

El detalle de los resultados se presenta en la tabla No.3, seis de los grupos de datos (síntomas leve y muy leve, faringitis, LDH, dímero $\mathrm{D}$ elevado, linfocitosis y Procalcitonina) presentan una heterogeneidad mayor al 75\%, y además la prueba de Egger no es aplicable en dos grupos, por que existe solo dos estudios con datos en cada uno de estos.

\section{Figura No.1}




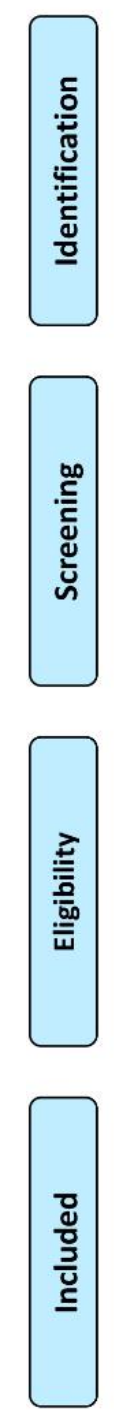
Registros identificados a través de
la búsqueda en la base de datos $(n=92)$
Registros adicionales identificados a través de otras fuentes $(n=0)$

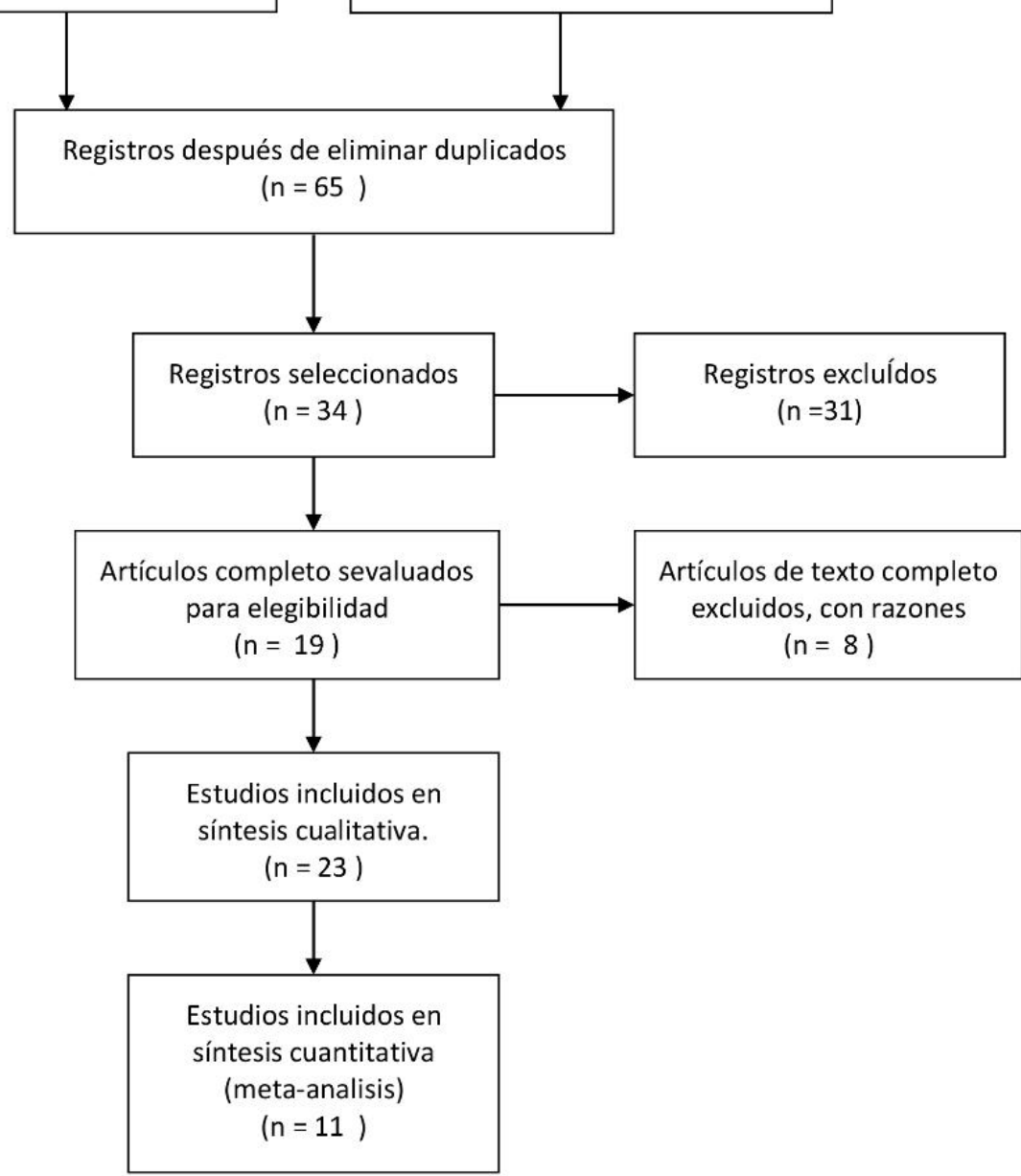

Figura 1. PRISMA 2009 Flow Diagram From: Moher D, Liberati $\Lambda$, T'tzlaff $J$, Altman DG, The PRISMA Group (2009). Preferred Reporting Items for Systematic Reviews and Meta-Analyses: The PRISMA Statement. PLoS Med 6(6): e1000097. doi:10.1371/journal.pmed1000097

For more information, visit www.prisma-statement.org.

Tomado y adaptado de: prisma-statement.org 
Tabla . 1 Distribución de los pacientes de acuerdo con el sexo y los grupos de edad en cada estudio

\begin{tabular}{|c|c|c|c|c|c|c|c|c|c|c|c|c|c|c|c|c|c|c|}
\hline Autor & \begin{tabular}{|l|} 
Promedio \\
edad \\
(Rango)
\end{tabular} & $\mathbf{n}$ & Edad 1 & $\%$ & Edad 2 & $\%$ & Edad 3 & $\%$ & Edad 4 & $\%$ & Edad 5 & $\%$ & M & $\%$ & $\mathbf{F}$ & $\%$ & Tipo de estudio & País \\
\hline Lu. X \& cols & $6.7 \mathrm{a}(1 \mathrm{~d}-15)$ & 171 & $<1 a=31$ & 18.1 & $1-5 \mathrm{a}=40$ & 23.4 & $\begin{array}{c}\text { 6- } 10 \mathrm{a} \\
=58\end{array}$ & 33.9 & $11-15 a=42$ & 24.6 & - & & 104 & 60.8 & 67 & 39.2 & Serie de casos & China \\
\hline Cai. J \& cols & $\begin{array}{l}74 \mathrm{~m} \\
(3-131)\end{array}$ & 10 & $<1 \mathrm{a}=2$ & 20 & $1-5 \mathrm{a}=2$ & 20 & $6-10 a=4$ & 40 & $11-15 a=2$ & 20 & - & & 4 & 40 & 6 & 60 & Serie de casos & China \\
\hline Chen. C \& cols. & $\begin{array}{l}7.33 \\
(1.5-17)\end{array}$ & 31 & $0-4 a=10$ & 32.2 & $5-9 a=13$ & 41.9 & $10-14 a=6$ & 19.4 & $15-17 \mathrm{a}=2$ & 6.5 & - & & 13 & 41.9 & 18 & 58.1 & Serie de casos & China \\
\hline Feng. K \& cols. & $7 a(4-14 a)$ & 15 & - & - & - & & - & - & - & - & - & & 5 & 33.3 & 10 & 66.7 & Serie de casos & China \\
\hline $\begin{array}{l}\text { Henry.B } \\
\text { \& Oliveira.M }\end{array}$ & $\begin{array}{l}10 \mathrm{a} \\
5-15 \mathrm{a})\end{array}$ & 82 & $0-4 a=16$ & 23.3 & $5-9 \mathrm{a}=18$ & 26.1 & $\begin{array}{l}10-14 \mathrm{a} \\
=12\end{array}$ & 17 & $15-19 \mathrm{a}=23$ & 33.3 & - & & 43 & 53.4 & 27 & 32.9 & $\begin{array}{l}\text { Observacional } \\
\text { analítico }\end{array}$ & varios \\
\hline Huijing. Ma \& cols & - & 50 & - & - & - & - & - & - & - & - & - & & 28 & 56 & 22 & 44 & Serie de casos & China \\
\hline Tang. A \& Cols & $6.9 a(1-13 a)$ & 26 & $1-3 a=5$ & 19 & $3-7 \mathrm{a}=8$ & 31 & $7-14 a=13$ & 50 & & - & - & & 17 & 65 & 9 & 35 & Serie de casos & China \\
\hline Dong . Y \& cols & $\begin{array}{l}7 \mathrm{a} \\
2-13)\end{array}$ & 731 & $<1 \mathrm{a}=86$ & 11.8 & $1-5 a=137$ & 18.7 & $\begin{array}{c}6-10 a= \\
171\end{array}$ & 23.4 & $11-15 a=180$ & 24.6 & $>15 a=157$ & 21.5 & 420 & 57.5 & 311 & 42.5 & $\begin{array}{l}\text { Observacional } \\
\text { analítico }\end{array}$ & China \\
\hline Xia. W \& cols & $\begin{array}{l}2 \text { a } 1,5 \mathrm{~m} \\
(1 \mathrm{~d}-14 \mathrm{a} 7 \mathrm{~m})\end{array}$ & 20 & $<1 \mathrm{~m}=3$ & 15 & $1 \mathrm{~m}-1 \mathrm{a}=6$ & 30 & $1-3 a=5$ & 25 & $3-6 a=3$ & 15 & $>6 a=3$ & 15 & 13 & 65 & 7 & 35 & Serie de casos & China \\
\hline Xu. Y \& cols & $\begin{array}{l}90 \mathrm{~m} \\
182 \mathrm{~m})\end{array}$ & 10 & $<1 \mathrm{a}=1$ & 10 & $1-5 a=3$ & 30 & $6-10 a=2$ & 20 & $11-15 a=3$ & 30 & - & - & 6 & 60 & 4 & 40 & Serie de casos & China \\
\hline Zhang, C \& cols & $\begin{array}{l}33 \mathrm{~m} \\
\text { (IQR 10-94.2) }\end{array}$ & 34 & - & - & - & - & - & - & - & - & - & - & 14 & 41.1 & 20 & 58.8 & Serie de casos & China \\
\hline
\end{tabular}

$\mathrm{a}=$ años, $\mathrm{m}=$ meses, $\mathrm{d}=$ días, $\mathrm{IQR}=$ intervalo intercuartil, $\mathrm{M}=$ masculino, $\mathrm{F}=$ femenino 
Tabla No.2 Distribución de acuerdo con las características clínicas.

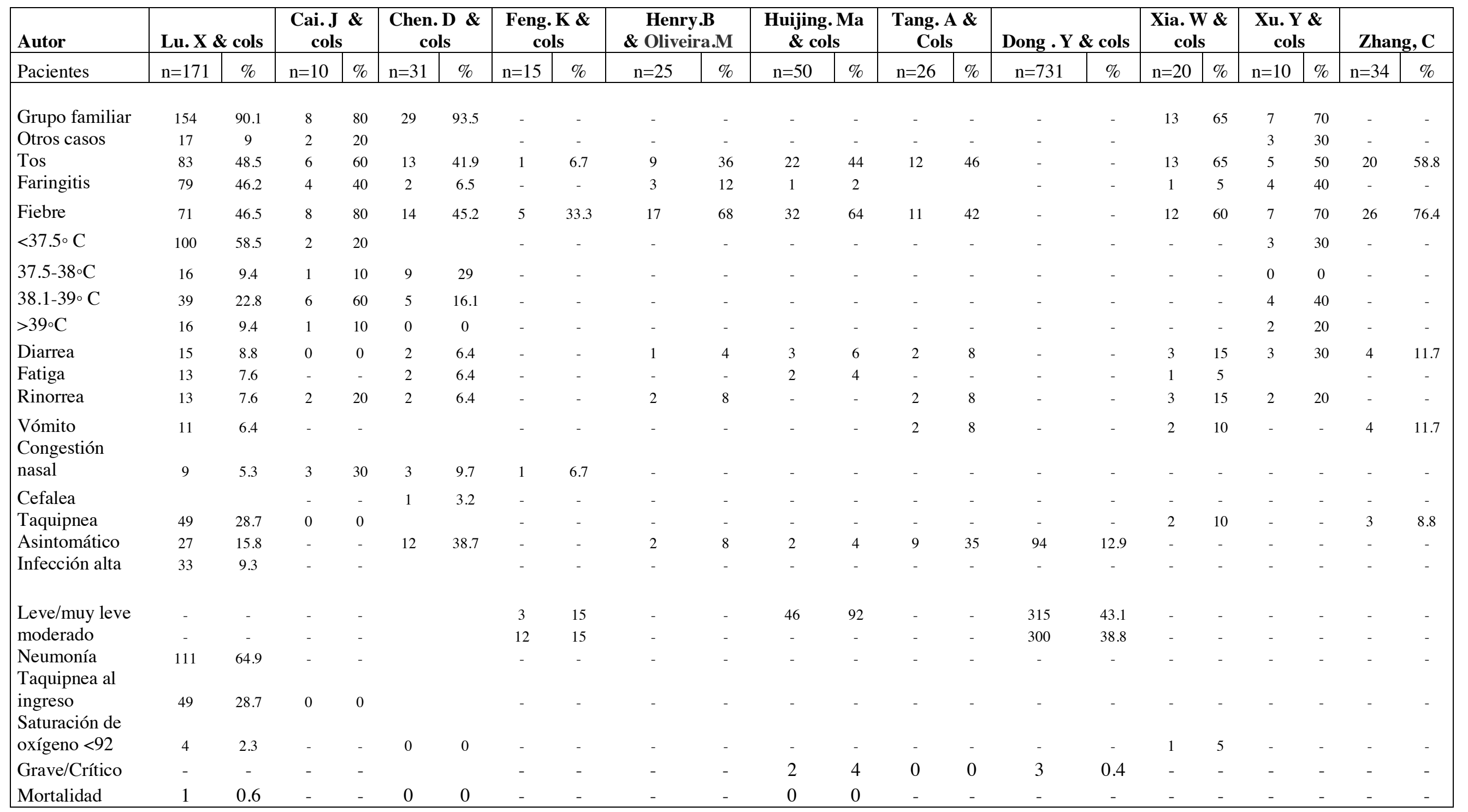

${ }^{2}$ Donde se contagio el paciente . T= Temperatura más alta del paciente durante su hospitalización. (-)no se reporta dato. 
Tabla No.3 Distribución de acuerdo con las características de laboratorio y radiología.

\begin{tabular}{|c|c|c|c|c|c|c|c|c|c|c|c|c|c|c|c|c|c|c|c|c|c|c|}
\hline \multirow{2}{*}{$\begin{array}{l}\text { Autor } \\
\text { Pacientes }\end{array}$} & \multicolumn{2}{|c|}{ Lu. X \& cols } & \multicolumn{2}{|c|}{$\begin{array}{c}\text { Cai. J \& } \\
\text { cols }\end{array}$} & \multicolumn{2}{|c|}{$\begin{array}{c}\text { Chen. D \& } \\
\text { cols }\end{array}$} & \multicolumn{2}{|c|}{$\begin{array}{c}\text { Feng. } K \& \\
\text { cols }\end{array}$} & \multicolumn{2}{|c|}{$\begin{array}{c}\text { Henry.B } \\
\text { \& Oliveira.M } \\
\end{array}$} & \multicolumn{2}{|c|}{$\begin{array}{c}\text { Huijing. } \\
\text { Ma \& cols }\end{array}$} & \multicolumn{2}{|c|}{$\begin{array}{c}\text { Tang. A \& } \\
\text { Cols }\end{array}$} & \multicolumn{2}{|c|}{$\begin{array}{c}\text { Dong . Y \& } \\
\text { cols }\end{array}$} & \multicolumn{2}{|c|}{$\begin{array}{c}\text { Xia. W \& } \\
\text { cols }\end{array}$} & \multicolumn{2}{|c|}{ Xu. Y \& cols } & \multicolumn{2}{|c|}{ Zhang, C } \\
\hline & $\mathrm{n}=171$ & $\%$ & $\mathrm{n}=10$ & $\%$ & $\mathrm{n}=31$ & $\%$ & $\mathrm{n}=15$ & $\%$ & $\mathrm{n}=25$ & $\%$ & $\mathrm{n}=50$ & $\%$ & $\mathrm{n}=26$ & $\%$ & $\mathrm{n}=731$ & $\%$ & $\mathrm{n}=20$ & $\%$ & $\mathrm{n}=10$ & $\%$ & $\mathrm{n}=34$ & $\%$ \\
\hline $\begin{array}{l}\text { Saturación de } \\
\text { oxígeno }<92\end{array}$ & 4 & 2.3 & - & - & 0 & 0 & - & - & - & - & - & - & - & - & - & - & 1 & 5 & - & - & - & - \\
\hline Leucocitosis & - & - & 3 & 30 & 1 & 3.2 & - & - & - & - & - & - & 4 & 15 & - & - & 2 & 10 & 0 & 0 & 0 & 0 \\
\hline Leucopenia & 45 & 26.3 & 1 & 10 & 12 & 38.7 & 8 & 53.2 & - & - & 19 & 38 & 13 & 50 & - & - & 4 & 20 & 3 & 33.3 & 0 & 0 \\
\hline Linfocitosis & - & - & - & - & 8 & 25.8 & - & - & - & - & 4 & 8 & 25 & 96 & - & - & 3 & 15 & 2 & 22.2 & 17 & 28 \\
\hline Linfopenia & 6 & .5 & 0 & 0 & 0 & 0 & - & - & - & - & 10 & 20 & 1 & 4 & - & - & 7 & 35 & 3 & 33.3 & 0 & 50 \\
\hline Trombocitopenia & - & - & - & - & 1 & 3.2 & - & - & - & - & - & - & - & - & - & - & - & - & - & - & - & - \\
\hline Procalcitonina & 105 & 64 & 0 & 0 & 1 & 3.2 & - & - & - & - & - & - & 0 & 0 & - & - & 16 & 80 & 5 & 55 & 0 & 0 \\
\hline PCR & 33 & 19.7 & 3 & 30 & 4 & 12.9 & - & - & - & - & 9 & 18 & 5 & 19 & - & - & 7 & 35 & 3 & 33.3 & - & - \\
\hline AST & 21 & 12.3 & 0 & 0 & & & - & - & - & - & - & - & 3 & 12 & - & - & - & - & 2 & 22.2 & - & - \\
\hline Dímero D elevado & 21 & 14.1 & 2 & 20 & & & - & - & - & - & - & - & - & - & - & - & - & - & 7 & 7.7 & - & - \\
\hline $\begin{array}{l}\text { LDH } \\
\text { Anormalidades }\end{array}$ & 0 & 0 & 3 & 30 & 12 & 38.7 & - & - & - & - & - & - & 12 & 46 & - & - & - & - & 2 & 22.2 & 28 & 82.3 \\
\hline radiológicas & 111 & 64.9 & 4 & 40 & 11 & 35.5 & 9 & 60 & - & - & 43 & 86 & 18 & 69 & - & - & 16 & 80 & 7 & 77.7 & 28 & 82.3 \\
\hline
\end{tabular}

$\mathrm{PCR}=$ Proteína $\mathrm{C}$ reactiva, $\mathrm{AST}=$ Aspartato amino transferasa, $\mathrm{ALT}=$ Alanima amino transferasa, $\mathrm{LDH}=\mathrm{Deshidrogenasa}$ láctica. Elevadas sobre el rango normal para la edad. 
Tabla 4. Resultados del Metaanálisis

\begin{tabular}{|c|c|c|c|c|c|c|c|}
\hline \multirow[b]{2}{*}{ Datos } & $\begin{array}{l}\text { Modelo } \\
\text { efectos fijo }\end{array}$ & & $\begin{array}{c}\text { Modelo } \\
\text { efectos } \\
\text { aleatorios }\end{array}$ & & $\begin{array}{c}\text { Heterogeneidad } \\
\% \mathrm{I}^{2}\end{array}$ & \multirow[b]{2}{*}{$\mathrm{p}$} & \multirow[b]{2}{*}{ Egger } \\
\hline & Proporción & IC $95 \%$ & Proporción & IC $95 \%$ & & & \\
\hline Masculino & 0.57 & $(0.54: 0.60)$ & 0.56 & $(0.51: 0.61)$ & 24.9 & 0.2 & 0.23 \\
\hline Asintomático & 0.12 & $(0.1: 0.15)$ & 0.15 & $(0.09: 0.22)$ & 59 & $<0.01$ & 0.44 \\
\hline Leve/ muy leve & 0.45 & $(0.41: 0.48)$ & 0.44 & $(0.25: 0.63)$ & 88 & $<0.01$ & $\mathrm{Na}$ \\
\hline Moderado & 0.41 & $(0.38: 0.45)$ & 0.41 & $(0.38: 0.45)$ & 0 & 0.51 & $\mathrm{Na}$ \\
\hline Fiebre & 0.52 & $(0.47: 0.57)$ & 0.56 & $(0.48: 0.65)$ & 66 & $<0.01$ & 0.12 \\
\hline Tos & 0.47 & $(0.42: 0.52)$ & 0.46 & $(0.39: 0.53)$ & 48 & 0.04 & 0.79 \\
\hline Diarrea & 0.07 & $(0.04: 0.1)$ & 0.07 & $(0.04: 0.1)$ & 3 & $<0.01$ & 0.29 \\
\hline Fatiga & 0.06 & $(0.03: 0.09)$ & 0.06 & $(0.03: 0.09)$ & 0 & 0.89 & 0.29 \\
\hline Faringitis & 0.26 & $(0.21: 0.31)$ & 0.18 & $(0.03: 0.39)$ & 92 & $<0.01$ & 0.77 \\
\hline Rinorrea & 0.07 & $(0.4: 0.11)$ & 0.07 & $(0.4: 0.11)$ & 0 & 0.62 & 0.22 \\
\hline Congestión & 0.05 & $(0.02: 0.09)$ & 0.08 & $(0.02: 0.17)$ & 49 & 0.12 & 0.15 \\
\hline ALT & 0.12 & $(0.08: 0.17)$ & 0.12 & $(0.08: 0.17)$ & 0 & 0.65 & 0.99 \\
\hline AST & 0.09 & $(0.02: 0.19)$ & 0.09 & $(0.02: 0.19)$ & 0 & 0.42 & 0.96 \\
\hline $\begin{array}{l}\text { Alteración } \\
\text { radiológica }\end{array}$ & 0.69 & $(0.64: 0.73)$ & 0.68 & $(0.56: 0.79)$ & 75 & $<0.01$ & 0.91 \\
\hline $\mathrm{LDH}$ & 0.53 & $(0.43: 0.62)$ & 0.45 & $(0.24: 0.7)$ & 81 & $<0.01$ & 0.26 \\
\hline $\begin{array}{l}\text { Dímero D } \\
\text { elevado }\end{array}$ & 0.13 & $(0.08: 0.19)$ & 0.32 & $(0.02: 0.73)$ & 89 & $<0.01$ & 0.23 \\
\hline Leucocitosis & 0.09 & $(0.04: 0.14)$ & 0.1 & $(0.04: 0.18)$ & 66 & 0.01 & 0.22 \\
\hline Leucopenia & 0.31 & $(0.26: 0.36)$ & 0.33 & $(0.25: 0.42)$ & 50 & 0.05 & 0.43 \\
\hline Linfopenia & 0.05 & $(0.03: 0.09)$ & 0.08 & $(0.03: 0.15)$ & 72 & 0.01 & 0.29 \\
\hline Linfocitosis & 0.09 & $(0.06: 0.13)$ & 0.27 & $(0.09: 0.49)$ & 95 & $<0.01$ & 0.29 \\
\hline PCR & 0.19 & $(0.15: 0.24)$ & 0.19 & $(0.15: 0.24)$ & 0 & 0.84 & 0.18 \\
\hline Procalcitonina & 0.43 & $(0.37: 0.5)$ & 0.26 & $(0.16: 0.46)$ & 93 & $<0.01$ & 0.25 \\
\hline
\end{tabular}

Tabla 4. Resultados del Metaanálisis

Heterogeneidad \% $\mathrm{I}^{2}=$ Estimación del porcentaje de Heterogeneidad en cada grupo de datos. $\mathrm{p}=\mathrm{p}$-valor del test de heterogeneidad.Egger $=$ valor $\mathrm{p}$ de la regresión linear de Egger 


\section{Discusión}

Las manifestaciones clínicas del COVID-19 son comparadas con los brotes de influenza estacional, donde la mayoría de las personas infectadas presentan síntomas leves, y las personas mayores o portadores de comorbilidades presentan mayor probabilidad de complicaciones. Se han establecido 3 patrones de presentación del cuadro clínico: 1)Leve con síntomas de tracto respiratorio superior, 2) Neumonía sin síndrome de dificultad respiratoria, y 3)Neumonía con síndrome de dificultad respiratoria, con una letalidad de aproximadamente $2 \% 1,18,19$, en este estudio se encontró que el $15 \%$ de pacientes fueron asitomáticos, ademas el 44 y $41 \%$, presentaron sintomas leves y moderados respectivamente, aunque estos últimos no son datos confiables por el grado de heterogeneidad que presentan, muy pocos niños presentaron condición grave o crítica y solo se reporta un fallecido, que fue precisamente un niño que además tenía otras comorbilidades.

La mayoría de contagios se produjeron en el núcleo familiar; el $56 \%$ de los afectados fueron de sexo masculino, resultado muy similar a lo referido en estudios realizados en adultos donde la proporción de hombres es todavía mayor, existe algunos aspectos de la fisiología humana donde se le atribuye al cromosoma $\mathrm{X}$ un rol protector ${ }^{1}$.

En adultos se ha estimado que la presencia de fiebre es del $89.1 \%$, la tos se presenta en el $72.2 \%{ }^{20}$, la intensidad de la tos estaría explicada por la afinidad del virus al receptor ECA2 actuando de manera similar a los inhibidores de la enzima convertidora de angiotensina (IECA), es decir no permite la degradación de bradicinina y sustancia $\mathrm{P}$ conocidos factores proinflamatorios que liberan prostaglandinas y oxido nítrico causantes de la tos ${ }^{4}$, en este estudio se encontró tos en el $46 \%$ de los pacientes y fiebre en el $56 \%$, 
estos menores porcentajes estarían explicados por el mayor número de pacientes menores de 19 años asintomáticos o con infección leve.

El patrón radiográfico caracterizado por múltiples sombras irregulares (vidrio esmerilado $)^{10,21}$ presente en el $96.6 \%$ de adultos ${ }^{20}$ y el $68 \%$ de niños de acuerdo con los hallazgos de este estudio, destaca que en ambos grupos la lesión pulmonar evidenciada por la radiología supera a las otras variables estudiadas. Este hallazgo puede explicarse por la capacidad de COVID-19 de interactuar con el receptor para la enzima convertidora de angiotensina humana (ECA2), la misma que se expresa en células epiteliales alveolares tipo I y con más intensidad en los neumocitos tipo II, provocando alteración del parénquima pulmonar por daño alveolar masivo secundario a descamación de los neumocitos, edema con infiltrado inflamatorio mononuclear, depósito de membranas sobre la superficie de intercambio gaseoso ${ }^{21}$, Es importante anotar que, a pesar del mecanismo patogénico que provoca el virus, no todos los pacientes muestran dificultad respiratoria ni hipoxemia. Además, hay pacientes con resultado positivo asintomáticos. ${ }^{22,4}$ La capa basal del epitelio escamoso no queratinizado de la mucosa nasal, oral y nasofaríngea, tambien son los tejidos diana del agente infeccioso ${ }^{4}$, el $7 \%$ de los pacientes presentaron rinorrea, el 5\% presentó congestión nasal por lo que no se podría considerar signos frecuentes en COVID-19.

A estos dos síntomas se suman el recuento normal o disminuido de glóbulos blancos, la linfopenia que es considerada un factor crítico asociado a la gravedad y mortalidad de la enfermedad en adultos. ${ }^{21}$ Entre los hallazgos inmunológicos se describe la lesión inmune severa con recuento de células $\mathrm{T}$ periféricas tanto $\mathrm{CD} 4$ como CD8 disminuidas, pero hiperactivadas con altas concentraciones de HLA-DR y gránulos citotóxicos ${ }^{21}$ Según reportes realizados en adultos la linfopenia es más frecuente pero está asociada a la gravedad del cuadro clínico y por tanto a un mayor índice de mortalidad ${ }^{21}$, en este 
estudio se encontró leucocitosis 9\%, leucopenia 31\%, linfopenia 5\% este hallazgo puede estar relacionado tanto con las características del sistema inmunológico, como con el bajo porcentaje de pacientes con cuadro clínico grave reportado en los estudios analizados.

Tanto el PCR (proteína C reactiva ) como la PCT (procalcitonina) se elevan en procesos inflamatorios-infecciosos, la procalcitonina es muy útil para la diferenciación de enfermedades infecciosas bacterianas graves, pero también se ha descrito aumento de sus valores en procesos virales ${ }^{23-25}$. Su elevación obedece a una respuesta inflamatoria sistémica, es decir mientras mas altos sean sus niveles la probabilidad de consecuencias graves de una infección son mayores, permitiendo entonces un mejor manejo de los pacientes. ${ }^{26}$ Se ha indicado que los resultados de las dos pruebas son normales en pacientes con cuadro clínico leve y que pueden estar normales o aumentadas en cuadros graves con sobreinfección bacteriana. ${ }^{27}$, en niños la PCR se presentó elevada en el $19 \%$.

Síntomas como diarrea y vómito pueden estar presentes, en este estudio se encontró 7\% de pacientes con diarrea, en los estudios se reporta presencia de los virus en las heces fecales de los niños hasta 30 días después del contagio ${ }^{8}$, sin embargo la transmisión fecaloral aún no se ha determinado. ${ }^{18}$

Igual que en infecciones por otros tipos de coronavirus se ha observado menor afectación de la población pediátrica, y además la presentación de síntomas leves o nulos, ${ }^{19,27}$ esto ocurre a pesar que los niños y jóvenes tiene mayor expresión del receptor ECA2, sugiriendo que este factor fisiológico no se encuentra relacionado con la gravedad de la infección, a diferencia del funcionamiento del sistema inmunológico, por tanto se ha propuesto la hipótesis de que las características inmunitarias propias de la edad constituyen un factor protector. ${ }^{4,28}$ Este patrón de presentación en niños y adolescentes 
ha llamado mucho la atención, puesto que estos grupos generalmente son los más afectados por las infecciones respiratorias virales. ${ }^{29}$ Se ha reportado que aquellos paciente que han requerido cuidados intensivos y ventilación mecánica eran además portadores de otras morbilidades ${ }^{23}$.

En conclusión; la presencia de anormalidades radiológicas es mas frecuente que los signos clínicos de tos y fiebre que se consideran los mas comunes, estos hallazgos pueden ser explicados por la afinidad del virus con las células del tracto respiratorio inferior, causando evidencia radiológica de lesion pulmonar aún en pacientes que no presentan cuadro clínico de neumonía, a diferencia del PCR que es considerado un factor de gravedad.En el hemograma, la leucopenia es mas frecuente, además existe una tendencia de mayor afectación en el sexo masculino. Hasta la fecha de revisión no pudimos encontrar estudios publicados de cohortes, caso control o ensayos controlados aleatorizados. Por lo que este metaanálisis está realizado con los mejores datos disponibles, con un riguroso análisis por lo que los datos válidos son altamente creíbles y servirán de base para futuras comparaciones acerca de las similitudes o diferenias que pueden presentarse ahora que la pandemia está en América.

Las limitaciones de este estudio son: a) todos los datos son retrospectivos, b) existe seis grupos de datos que presentaron heterogeneidad mayor a $75 \%$ por lo que sus resultados deben ser considerados con cautela, c) no todos los estudios presentaban las variables estudiadas, y el número de observaciones no fue constante, d) la mayoría de los datos correspoden a pacientes chinos. Considerando estas limitaciones, los resultados de este estudio deben ser contrastados, con estudios prospectivos controlados, con mayor número de pacientes y un diseño mas estricto. 


\section{Financiación}

El presente trabajo así como la investigación precedente no han recibido financiación alguna para su elaboración.

\section{Conflictos de interés}

Los autores declaran no tener ningún conflicto de intereses.

\section{Contribución de los autores}

Santiago Vasco-Morales y Paola Toapanta-Pinta. selección y análisis de documentos, referencias bibliográficas, y preparación del manuscrito.

Cristhian Vasco-Toapanta. Búsqueda de bibliografía, extracción y análisis estadístico de los datos

\section{Bibliografía:}

1. Palacios Cruz M, Santos E, Velázquez Cervantes MA, León Juárez M. COVID19, una emergencia de salud pública mundial. Rev Clínica Española. 2020;

2. Armijos RJT, Romero JIQ, Aquino PAB, Perero GJL. Importancia en el cuidado de enfermedades respiratorias en niños menores de 5 años. RECIAMUC. 2018;2:229-44.

3. Iñiguez F, Sánchez I. Desarrollo pulmonar. Neumonol Pediatríca. 2008;3:148-55.

4. Sun ML, Yang JM, Sun YP, Su GH. Inhibitors of RAS might be a good choice for the therapy of COVID-19 pneumonia. Zhonghua jie he he hu xi za zhi= Zhonghua jiehe he huxi zazhi= Chinese J Tuberc Respir Dis. 2020;43:E014.

5. R Core Team. R: A language and environment for statistical computing [Internet]. Vienna, Austria.: R Foundation for Statistical Computing; 2019. Available from: https://www.r-project.org/. 
6. Balduzzi S, Rücker G, Schwarzer G. How to perform a meta-analysis with R: a practical tutorial. Evid Based Ment Heal [Internet]. 2019;22:153-60. Available from: http://ebmh.bmj.com/lookup/doi/10.1136/ebmental-2019-300117

7. Lu X, Zhang L, Du H, Zhang J, Li YY, Qu J, et al. SARS-CoV-2 Infection in Children. N Engl J Med [Internet]. 2020; Available from: https://doi.org/10.1056/NEJMc2005073

8. Cai J, Xu J, Lin D, Yang zhi, Xu L, Qu Z, et al. A Case Series of children with 2019 novel coronavirus infection: clinical and epidemiological features. Clin Infect Dis [Internet]. 2020; Available from: https://doi.org/10.1093/cid/ciaa198

9. Chen C, Cao M, Peng L, Guo X, Yang F, Wu W, et al. Coronavirus Disease-19 Among Children Outside Wuhan, China. China (2/25/2020). 2020;

10. Feng K, Yun YX, Wang XF, Yang GD, Zheng YJ, Lin CM, et al. [Analysis of CT features of 15 Children with 2019 novel coronavirus infection]. Zhonghua er ke za zhi = Chinese J Pediatr [Internet]. 2020 [cited 2020 Mar 22];58:E007. Available from: http://www.ncbi.nlm.nih.gov/pubmed/32061200

11. Henry BM, Oliveira MHS. Preliminary epidemiological analysis on children and adolescents with novel coronavirus disease 2019 outside Hubei Province, China: an observational study utilizing crowdsourced data. medRxiv. 2020;

12. Ma H and H, AndTian J, AndZhou J, Li X and, Laws H and, Wesemann MTA, et al. The Lancet Visualizing the novel coronavirus ( COVID-19) in children : What we learn from patients at Wuhan Children's Hospital. Lancet [Internet]. 2020;22. Available from: https://papers.ssrn.com/sol3/papers.cfm?abstract_id=3550012

13. Tang A, Xu W, Chen P, Li G, Liu Y, Liu L. A retrospective study of the clinical characteristics of COVID-19 infection in 26 children. medRxiv. 2020;

14. Dong Y, Mo X, Hu Y, Qi X, Jiang F, Jiang Z, et al. Epidemiological characteristics 
of 2143 pediatric patients with 2019 coronavirus disease in China. Pediatrics. 2020;

15. Xia W, Shao J, Guo Y, Peng X, Li Z, Hu D. Clinical and CT features in pediatric patients with COVID-19 infection: Different points from adults. Pediatr Pulmonol [Internet]. 2020 [cited 2020 Apr 1];ppul.24718. Available from: https://onlinelibrary.wiley.com/doi/abs/10.1002/ppul.24718

16. Xu Y, Li X, Zhu B, Liang H, Fang C, Gong Y, et al. Characteristics of pediatric SARS-CoV-2 infection and potential evidence for persistent fecal viral shedding. Nat Med. 2020;1-4.

17. Zhang C, Gu J, Chen Q, Deng N, Li J, Huang L, et al. Clinical Characteristics of 34 Children with Coronavirus Disease-2019 in the West of China: a Multiplecenter Case Series. medRxiv [Internet]. 2020 [cited 2020 Apr 1];2020.03.12.20034686. Available from: https://www.medrxiv.org/content/10.1101/2020.03.12.20034686v1

18. Heymann DL, Shindo N. COVID-19: what is next for public health? Lancet. 2020;395:542-5.

19. Deng S-Q, Peng H-J. Characteristics of and Public Health Responses to the Coronavirus Disease 2019 Outbreak in China. J Clin Med. 2020;9:575.

20. Sun P, Qie S, Liu Z, Ren J, Li K, Xi J. Clinical characteristics of 50466 hospitalized patients with 2019-nCoV infection. J Med Virol [Internet]. 2020;jmv.25735. Available from: https://onlinelibrary.wiley.com/doi/abs/10.1002/jmv.25735

21. Xu Z, Shi L, Wang Y, Zhang J, Huang L, Zhang C, et al. Pathological findings of COVID-19 associated with acute respiratory distress syndrome. Lancet Respir Med. 2020; 
22. Liu Y, Gayle AA, Wilder-Smith A, Rocklöv J. The reproductive number of COVID-19 is higher compared to SARS coronavirus. J Travel Med. 2020;

23. Lu X, Zhang L, Du H, Zhang J, Li YY, Qu J, et al. SARS-CoV-2 Infection in Children. N Engl J Med. 2020;

24. Kong W, Agarwal PP. Chest imaging appearance of COVID-19 infection. Radiol Cardiothorac Imaging. 2020;2:e200028.

25. Fang Y, Zhang H, Xie J, Lin M, Ying L, Pang P, et al. Sensitivity of chest CT for COVID-19: comparison to RT-PCR. Radiology. 2020;200432.

26. González Montoya C, Hernández Luna A, Villalobos Silva J, Aguirre Sánchez J, Franco Granillo J. Utilidad de procalcitonina como marcador diagnóstico temprano en choque séptico. Rev la Asoc Med Crítica y Ter Intensiva. 2009;XXIII:211-7.

27. Calvo C, López-Hortelano MG, de Carlos Vicente JC, Martínez JLV, de trabajo de la Asociación G, Ramos JT, et al. Recomendaciones sobre el manejo clínico de la infección por el «nuevo coronavirus»SARS-CoV2. Grupo de trabajo de la Asociación Española de Pediatría (AEP). In: Anales de Pediatría. Elsevier; 2020.

28. Kam K, Yung CF, Cui L, Lin Tzer Pin R, Mak TM, Maiwald M, et al. A well infant with coronavirus disease 2019 (COVID-19) with high viral load. Clin Infect Dis. 2020;

29. de Carvalho AP. Novo coronavírus (COVID-19). 2020. 